\title{
Color-dipole picture versus hard pomeron in deep inelastic scattering
}

\author{
Dieter Schildknecht $\odot$ \\ Fakultät für Physik, Universität Bielefeld D-33501 Bielefeld, Germany \\ and Max-Planck-Institut für Physik (Werner-Heisenberg-Institut), \\ Föhringer Ring 6, D-80805 München, Germany
}

(Received 23 November 2020; accepted 9 June 2021; published 9 July 2021)

\begin{abstract}
For photon virtualities of $Q^{2} \gtrsim 20 \mathrm{GeV}^{2}$, the results of the (hard Pomeron) fit in the tensor-Pomeron model in terms of the variables, $Q^{2}$, and the virtual-photon-proton energy squared, $W^{2}$, provide empirical evidence for the validity of the color dipole picture (CDP). Consistency of the CDP with the perturbative QCD (pQCD) improved parton model implies the prediction of $C_{2}=\epsilon_{0} \cong 0.30$ for the exponent $C_{2}$ of the energy-squared dependence in agreement with the results of the fits. For $Q^{2} \lesssim 20 \mathrm{GeV}^{2}$, the CDP yields a parameter-free smooth transition from $Q^{2} \gtrsim 20 \mathrm{GeV}^{2}$ to $Q^{2}=0$ photoproduction, in distinction from the tensor-Pomeron model that relies on the additional parameter quantifying the intercept of the soft-Pomeron trajectory.
\end{abstract}

DOI: 10.1103/PhysRevD.104.014009

\section{INTRODUCTION}

The recent article [1] by Britzger et al. revives the twoPomeron-plus-Reggeon approach from Ref. [2] by introducing tensor couplings of the hard Pomeron, $\mathbb{P}_{0}$, the soft Pomeron, $\mathbb{P}_{1}$, and the $f_{2 R}$ Reggeon at the photon and proton vertices, $\gamma^{*} \gamma^{*}\left(\mathbb{P}_{0}, \mathbb{P}_{1}, f_{2 R}\right)$ and $p p\left(\mathbb{P}_{0}, \mathbb{P}_{1}, f_{2 R}\right)$, to fit the experimental data on deep-inelastic lepton-nucleon scattering (DIS) at low values of the Bjorken variable $x \simeq Q^{2} / W^{2}<0.01$, including the $Q^{2}=0$ photoproduction limit.

Regge theory, by generalization from hadronic interactions, assumes a power-law $W$ dependence for the photoabsorption cross section, as $\left(W^{2}\right)^{\alpha_{j}(0)-1}$, where the three [2] Regge intercepts, $\alpha_{0}(0)=1+\epsilon_{0}, \alpha_{1}(0)=1+\epsilon_{1}$ and $\alpha_{2}(0)$, are free parameters to be extracted from a fit to the experimental data. The form of the $Q^{2}$ dependence of the $\gamma^{*} \gamma^{*}\left(\mathbb{P}_{0}, \mathbb{P}_{1}, f_{2 R}\right)$ vertex contributions is not being fixed by Regge theory; in Ref. [1], the $Q^{2}$ dependence is modelindependently parametrized by smooth functions in terms of splines. Altogether the fit [1] contains 25 free parameters, namely the three Regge intercepts, five parameters for the $\gamma^{*} \gamma^{*}\left(\mathbb{P}_{0}, \mathbb{P}_{1}, f_{2 R}\right)$ couplings at $Q^{2}=0$, and 17 parameters for a model-independent description of the $Q^{2}$ dependence in terms of spline functions.

\footnotetext{
${ }^{1}$ In standard notation, $Q^{2}$ and $W^{2}$ denote the photon virtuality and the square of the photon-proton center-of-mass energy.

Published by the American Physical Society under the terms of the Creative Commons Attribution 4.0 International license. Further distribution of this work must maintain attribution to the author(s) and the published article's title, journal citation, and DOI. Funded by SCOAP ${ }^{3}$.
}

In the present article, we confront the interpretation of the DIS experimental data in Regge theory with their representation in the color-dipole picture (CDP). We proceed in three steps. In a first step, we concentrate on the region of large $Q^{2} \gtrsim 20 \mathrm{GeV}^{2}$, in Regge theory associated with the hard Pomeron $\mathbb{P}_{0}$ with intercept $\alpha_{0}(0)$. In the second step, we show that consistency of the CDP and the perturbative-QCD-improved parton model implies a successful prediction for the numerical value of the exponent of the $W^{2}$ dependence, $\epsilon_{0}=C_{2} \cong 0.30$. In the third step, we consider the transition to low $Q^{2}$, including the $Q^{2}=0$ limit of photoproduction, in Regge theory associated with the soft Pomeron with intercept $\alpha_{1}(0)$.

It will turn out that the model-independent 17-parameter fit of the $Q^{2}$ dependence in the tensor-Pomeron approach for $Q^{2} \gtrsim 20 \mathrm{GeV}^{2}$ leads to, and confirms, the parameterfree prediction of the $Q^{2}$ dependence of the CDP, wherein the photoabsorption cross section, $\sigma_{\gamma^{*}}\left(W^{2}, Q^{2}\right)$, depends on the low-x scaling variable $\eta^{-1}=\Lambda_{\text {sat }}^{2}\left(W^{2}\right)\left(Q^{2}+m_{0}^{2}\right)^{-1}$, i.e., $\sigma_{\gamma^{*} p}\left(W^{2}, Q^{2}\right)=\sigma_{\gamma^{*} p}\left(\eta\left(W^{2}, Q^{2}\right)\right)$, with $\Lambda_{\text {sat }}^{2}\left(W^{2}\right) \propto$ $\left(W^{2}\right)^{C_{2}}$ and $C_{2}=\epsilon_{0}$. Specifically, for $Q^{2} \gtrsim 20 \mathrm{GeV}^{2}$, in the CDP, $\sigma_{\gamma^{*} p} \propto \eta^{-1}=\Lambda_{\text {sat }}^{2}\left(W^{2}\right) / Q^{2}$, as confirmed by the tensor-Pomeron-fit result.

\section{LARGE VALUES OF $Q^{2} \gtrsim 20 \mathrm{GeV}^{2}$}

For large values of $Q^{2}$, the total photoabsorption cross section in the Regge approach is determined [1] by the hard-Pomeron contribution. According to the formula (6.1) in Ref. [1], the fit led to the result,

$$
\sigma_{\gamma^{*} p}\left(W^{2}, Q^{2}\right) \propto \hat{b}_{0}\left(Q^{2}\right)\left(W^{2}\right)^{\epsilon_{0}},
$$


where

$$
\epsilon_{0}=0.3008\left(\begin{array}{c}
+73 \\
-84
\end{array}\right) \cong 0.30 \pm 0.1
$$

and, with $\eta_{0}=0.967(73) \cong 1.0$,

$$
\hat{b}_{0}\left(Q^{2}\right)=\frac{1}{\left(Q^{2}\right)^{\eta_{0}}} \cong \frac{1}{Q^{2}} .
$$

According to (1) and (3), in the tensor-Pomeron model, for $Q^{2} \gtrsim 20 \mathrm{GV}^{2}$, the photoabsorption cross section fulfills the proportionality [1],

$$
\sigma_{\gamma^{*} p}\left(W^{2}, Q^{2}\right) \propto \frac{\left(W^{2}\right)^{\epsilon_{0}}}{Q^{2}},
$$

with $\epsilon_{0} \cong 0.30$ from (2).

In Ref. [3], in a phenomenological fit to the photoabsorption experimental data, for $x \lesssim 0.1$ and $0 \leq Q^{2} \lesssim$ $1000 \mathrm{GeV}^{2}$, technically by assuming a piecewise linear functional dependence of the cross section on the variable $\eta\left(W^{2}, Q^{2}\right)$ introduced [3] via

$$
\frac{1}{\eta\left(W^{2} Q^{2}\right)}=\frac{\Lambda_{\mathrm{sat}}^{2}\left(W^{2}\right)}{Q^{2}+m_{0}^{2}}
$$

where

$$
\Lambda_{\text {sat }}^{2}\left(W^{2}\right) \propto\left(W^{2}\right)^{C_{2}},
$$

we found

$$
\sigma_{\gamma^{*} p}\left(W^{2}, Q^{2}\right)=\sigma_{\gamma^{*} p}\left(\eta\left(W^{2}, Q^{2}\right)\right) .
$$

According to our fit, the photoabsorption cross section, including its $Q^{2}=0$ limit, only depends on the single low-x scaling variable $\eta\left(W^{2}, Q^{2}\right)$, rather than $W^{2}$ and $Q^{2}$ separately. The fit gave [3]

$$
C_{2}=0.28 \pm 0.06
$$

for the exponent $C_{2}$ in (6), as well as $m_{0}^{2}=0.125 \pm$ $0.027 \mathrm{GeV}^{2}$ for the mass parameter $m_{0}$, with $m_{0}^{2}<m_{\rho}^{2}$, as expected, where $m_{\rho}$ denotes the $\rho$-meson mass.

By inspection of the graphical representation of the experimental data for the photoabsorption cross section as a function of $\eta\left(W^{2}, Q^{2}\right)$, one reads off an explicit functional dependence that is approximately given by [3]

$$
\begin{gathered}
\sigma_{\gamma^{*} p}\left(W^{2}, Q^{2}\right)=\sigma_{\gamma^{*} p}\left(\eta\left(W^{2}, Q^{2}\right)\right) \propto \frac{1}{\eta\left(W^{2}, Q^{2}\right)}=\frac{\left(W^{2}\right)^{C_{2}}}{Q^{2}}, \\
\left(Q^{2}+m_{0}^{2} \gg \Lambda_{\mathrm{sat}}^{2}\left(W^{2}\right)\right),
\end{gathered}
$$

where $\Lambda_{\text {sat }}^{2}\left(W^{2}\right) \lesssim 7 \mathrm{GeV}^{2}$ in the energy range, where experimental data are available.

Comparison of (9) with (4), and of (8) with (2), reveals that for $Q^{2} \gtrsim 20 \mathrm{GeV}^{2}$ the tensor-Pomeron fit (1) from Ref. [1] confirms the result of the phenomenological fit (9) of $\sigma_{\gamma^{*} p}\left(W^{2}, Q^{2}\right) \propto 1 / \eta\left(W^{2}, Q^{2}\right)$ from Ref. [3].

A comment on the two-Pomeron fit of Ref. [2] is appropriate. In distinction from the model-independent fit of the $Q^{2}$ dependence in Ref. [1], the $Q^{2}$ dependence of the photoabsorption cross section in Ref. [2] is described by parameter-dependent analytic expressions in $Q^{2}$. The fit gave [2] an intercept of $\alpha_{0} \cong 1.4$ or

$$
\epsilon_{0} \cong 0.40
$$

significantly different from (2).

It is gratifying that the result of the tensor-Pomeron fit [1] for $Q^{2} \gtrsim 20 \mathrm{GeV}^{2}$, with $\epsilon_{0} \cong 0.30$, supports and confirms the fit in Ref. [3]. The tensor-Pomeron fit, under the restriction to $Q^{2} \gtrsim 20 \mathrm{GeV}^{2}$, has rediscovered the empirical scaling law [3] of $\sigma_{\gamma^{*} p}\left(W^{2}, Q^{2}\right)=\sigma_{\gamma^{*} p}\left(\eta\left(W^{2}, Q^{2}\right)\right)$.

Scaling in $\eta\left(W^{2}, Q^{2}\right)$, including the observed specific functional dependence (9) of $\sigma_{\gamma^{*} p}\left(W^{2}, Q^{2}\right) \propto$ $1 / \eta\left(W^{2}, Q^{2}\right)=\Lambda_{\text {sat }}^{2}\left(W^{2}\right) / Q^{2}$, valid for sufficiently large $Q^{2}$, is a consequence of the color-dipole picture [3]. For clarity and completeness, we elaborate on this essential point in detail.

The color dipole picture (CDP) of deep inelastic scattering (DIS) at low $\mathrm{x}$ is based on the transverse position-space representation,

$$
\begin{aligned}
\sigma_{\gamma_{L, T}^{*}}\left(W^{2}, Q^{2}\right)= & \int d z \int d^{2} \vec{r}_{\perp}\left|\psi_{L, T}\left(\vec{r}_{\perp}, z(1-z), Q^{2}\right)\right|^{2} \\
& \times \sigma_{(q \bar{q}) p}\left(\vec{r}_{\perp}^{2}, z(1-z), W^{2}\right)
\end{aligned}
$$

combined with the interaction of a $q \bar{q}$ color dipole with the color field in the nucleon [4],

$$
\begin{aligned}
\sigma_{(q \bar{q}) p}\left(\vec{r}_{\perp}^{2}, z(1-z), W^{2}\right)= & \int d^{2} \vec{l}_{\perp} \tilde{\sigma}\left(\vec{l}_{\perp}^{2}, z(1-z), W^{2}\right) \\
& \times\left(1-e^{-\vec{l}_{\perp} \vec{r}_{\perp}}\right) .
\end{aligned}
$$

In (11) and (12), $\vec{r}_{\perp}$ denotes the transverse interquark distance, $z(1-z)$ with $0 \leq z \leq 1$ describes the quarkantiquark $(q \bar{q})$ configuration and $\vec{l}_{\perp}$ the transverse gluon momentum absorbed by the $q \bar{q}$ state.

The representation (11), including the $(q \bar{q}) p$ interaction (12), follows [4] from off diagonal generalized vector dominance (GVD) [5] upon taking into account the internal structure of the vector states, $V \equiv(q \bar{q})^{J=1}$, in the $\gamma^{*} \rightarrow V$ transition, and transforming from momentum space to transverse position space. The minus sign in front of the exponential in (12), in the pre-QCD era originated $[5,4]$ 
from the required consistency [5] of scaling behavior in the timelike region of $e^{+} e^{-}$-annihilation, $e^{+} e^{-} \rightarrow$ hadrons, and in the spacelike region of deep inelastic scattering (DIS) of $e^{-} p \rightarrow e^{-}+$hadrons.

In QCD, the $(q \bar{q})^{J=1}$ states act as color-dipole states, thus recognizing the interaction (12) as a consequence of the color-dipole nature of the $(q \bar{q})^{J=1}$ states. In the limit of vanishing dipole size, $\vec{r}_{\perp}^{2} \rightarrow 0$, a $(q \bar{q})$-color-dipole state acts as a color-neutral object of vanishing interaction with the color field in the proton ("color transparency"), while for a large dipole size, $\vec{r}_{\perp}^{2} \rightarrow \infty$, the cross section has to remain finite and becomes hadronlike ("saturation"), thus leading to (12). A specific ansatz for the $(q \bar{q}) p$ cross section fulfilling the requirements of color transparency and saturation is mentioned in (28) below.

One observes that perturbative QCD ( $\mathrm{pQCD}$ ) has not been mentioned in arriving at the interaction cross section (12). In pQCD, the form of the interaction (12) is understood as a consequence of the color-gauge-invariant interaction of the $(q \bar{q})$ dipole with the color field in the nucleon via exchange of (at least) two gluons. The form of the position-space representation (11) with (12) from off diagonal GVD, from the point of view of pQCD is accordingly recognized as a consequence of the gaugeinvariant interaction of a $(q \bar{q})^{J=1}$-color-dipole state with the gluon field in the proton. The representation of the photoabsorption cross section in (11) and (12) is nevertheless a nonperturbative ${ }^{2}$ one; otherwise, it would not include and contain the smooth transition to the $Q^{2}=0$ photoproduction cross section.

It is the structure of the $(q \bar{q}) p$-interaction amplitude contained in (12) that is responsible for the CDP prediction of the $1 / Q^{2}$ dependence of the photoabsorption cross section, $\sigma_{\gamma^{*} p} \propto 1 / Q^{2}$ [given in (17) below], that, according to the fit result (1) with (3), is confirmed by the experimental data.

Upon introducing the variables $\vec{r}^{\prime}=\sqrt{z(1-z)} \vec{r}_{\perp}$ and $\vec{l}_{\perp}^{\prime}=\vec{l}_{\perp} / \sqrt{z(1-z)}$, and the cross sections for longitudinally and transversely polarized $(q \bar{q})_{L, T}^{J=1}(J=1$, vector $)$ states, for the color-dipole-proton cross section (12), one indeed finds $[3,7]$ the two limiting cases of "color transparency" for $\vec{r}_{\perp}^{2} \rightarrow 0$,

$$
\begin{aligned}
\sigma_{(q \bar{q})_{L, T}^{J=1} p}\left(r_{\perp}^{\prime 2}, W^{2}\right)= & \frac{1}{4} \pi \vec{r}_{\perp}^{2} \int d \vec{l}_{\perp}^{\prime 2} \vec{l}_{\perp}^{\prime 2} \bar{\sigma}_{(q \bar{q})_{L}^{J=1} p}\left(\vec{l}_{\perp}^{2}, W^{2}\right) \\
& \times\left\{\begin{array}{l}
1, \\
\rho_{W}
\end{array} \quad\left(\vec{r}_{\perp}^{2} \ll \frac{1}{\vec{l}_{\perp \mathrm{Max}}^{\prime 2}\left(W^{2}\right)}\right),\right.
\end{aligned}
$$

\footnotetext{
${ }^{2}$ In Ref. [6], "Towards a Non-Perturbative Derivation of the CDP", in a very detailed field-theoretic analysis, the form of the photoabsorption cross section (11) was reestablished, by assumption excluding a dependence of the dipole cross section on $z(1-z)$ in (11). The treatment in Ref. [6], rederiving (11), while not incorporating color transparency and saturation, misses an essential element of the CDP.
}

where

$$
\rho_{W} \equiv \frac{\int d \overrightarrow{l_{\perp}} \vec{l}_{\perp}^{2} \bar{\sigma}_{(q \bar{q})_{T}^{J=1} p}\left(\vec{l}_{\perp}^{2}, W^{2}\right)}{\int d \vec{l}_{\perp}^{\prime 2} \vec{l}_{\perp}^{2} \bar{\sigma}_{(q \bar{q})_{L}^{J=1} p}\left(\vec{l}_{\perp}^{2}, W^{2}\right)},
$$

and "saturation" for $\vec{r}_{\perp}^{2} \rightarrow \infty$,

$$
\begin{aligned}
& \sigma_{(q \bar{q})_{L, T}^{J=1} p}\left(r_{\perp}^{\prime 2}, W^{2}\right) \\
& \quad=\pi \int d \vec{l}_{\perp}^{2} \bar{\sigma}_{(q \bar{q})_{L, T}^{J=1} p}\left(\vec{l}^{\prime 2}, W^{2}\right) \\
& \quad \equiv \sigma_{L, T}^{(\infty)}\left(W^{2}\right), \quad\left(\vec{r}_{\perp}^{\prime 2} \gg \frac{1}{\vec{l}_{\perp \operatorname{Max}}^{\prime 2}\left(W^{2}\right)}\right) .
\end{aligned}
$$

In (13) and (15), $\vec{l}_{\perp \text { Max }}^{2}\left(W^{2}\right)$ denotes the upper limit of the range of integration over $d \vec{l}_{\perp}^{2}$ that yields the dominant contribution to the integrals. The parameter $\rho_{W}$ in (14), where $W$ indicates a potential dependence of $\rho=\rho_{W}$ on the energy $W$, determines the ratio of (the first moment of) the cross sections for the scattering of transversely (helicity \pm 1 ) and longitudinally (helicity 0) polarized $(q \bar{q})_{L, T}^{J=1}$ states on the proton. The deviation from $\rho_{W}=1$ quantifies the deviation of the $(q \bar{q})^{J=1}$-proton cross section from helicity independence of $(q \bar{q})_{\left.L_{\infty}\right)}^{J=1} p=(q \bar{q})_{T}^{J=1} p$.

The cross section $\sigma_{L, T}^{\infty}\left(W^{2}\right)$ in (15) is a purely hadronic quantity that fulfills hadronic unitarity, and it is at most weakly, logarithmically, dependent on the energy $W$.

The photoproduction cross section, as a consequence of the explicit form of the "photon wave function" $\psi_{L, T}\left(\vec{r}_{\perp}, z(1-z), Q^{2}\right)$ in (11), for sufficiently large $Q^{2} \gg 0$, receives contributions from only a finite range of $\vec{r}_{\perp}^{2}$ that is restricted by $\vec{r}_{\perp}^{2} \leq 1 / Q^{2}$. For given large energy $W$ and sufficiently large $Q^{2}$, such that $1 / Q^{2} \ll$ $1 / \vec{l}_{\perp \text { Max }}^{2}\left(W^{2}\right)$, the photoabsorption cross section (11) is determined by the color-dipole cross section for $\vec{r}_{\perp}^{2} \ll$ $1 / \vec{l}_{\perp \text { Max }}^{2}\left(W^{2}\right)$, i.e., in the color-transparency limit. Introducing the first moment of the distribution in $\vec{l}_{\perp}^{2}$ for $(q \bar{q})_{L}^{J=1} p$ scattering,

$$
\Lambda_{\text {sat }}^{2}\left(W^{2}\right) \equiv \frac{\int d \vec{l}_{\perp}^{2} \vec{l}_{\perp}^{2} \bar{\sigma}_{(q \bar{q})_{L}^{J=1} p}\left(\vec{l}_{\perp}^{2}, W^{2}\right)}{\int d \vec{l}_{\perp}^{\prime 2} \bar{\sigma}_{(q \bar{q})_{L}^{J=1} p}\left(\vec{l}_{\perp}^{2}, W^{2}\right)},
$$

the photoabsorption cross section (11), upon inserting (13) and employing (14) and (15), becomes [3,7]

$$
\sigma_{\gamma_{L, T}^{*} p}\left(W^{2}, Q^{2}\right) \propto \sigma_{L}^{(\infty)}\left(W^{2}\right) \frac{\Lambda_{\mathrm{sat}}^{2}\left(W^{2}\right)}{Q^{2}}\left\{\begin{array}{l}
1, \\
2 \rho_{W}
\end{array} .\right.
$$

With the approximation of $\sigma_{L}^{(\infty)}\left(W^{2}\right) \cong$ const, and $\rho_{W}=$ $\rho=$ const due to invariance under Lorentz boosts, and specifying $\Lambda_{\text {sat }}^{2}\left(W^{2}\right) \propto\left(W^{2}\right)^{C_{2}}$, we indeed recognize the result of the phenomenological fit (9) [that agrees with the 
tensor-Pomeron fit (1)] as a consequence of the CDP (11) and (12). The energy dependence of the fit (9), as $\Lambda_{\text {sat }}^{2}\left(W^{2}\right) \propto\left(W^{2}\right)^{C_{2}}$, according to (17) with (16) is recognized as the energy dependence of the effective transverse momentum of the gluon absorbed by the color-dipole $q \bar{q}$ state in the $\vec{r}_{\perp}^{2} \rightarrow 0$ limit.

While the fitted large- $Q^{2}$ energy dependence (1) of the photoabsorption cross section in the tensor-Pomeron model, as $\left(W^{2}\right)^{\epsilon_{0}}$, is consistent with the energy dependence (9) of the CDP, as $\left(W^{2}\right)^{C_{2}}$, its interpretation [1] is entirely different. The fitted energy dependence for $Q^{2} \gtrsim 20 \mathrm{GeV}^{2}$, in the tensor-Pomeron model is interpreted as evidence for the exchange of a hypothetical "hard-Pomeron trajectory" with intercept $\alpha_{0}(0)=1+\epsilon_{0}$, independent empirical evidence for this conjectured trajectory being lacking. Independently of this interpretation, the agreement of the fit (1) with the fit (9) now explicitly being recognized as a consequence of the CDP, explicitly confirms our above conclusion that the fit (1) provides additional empirical support for the previously empirically established validity [3] of the CDP of DIS at low $x \lesssim 0.1$.

\section{CONSISTENCY OF PQCD WITH THE CDP AND THE NUMERICAL VALUE OF $\boldsymbol{C}_{\mathbf{2}} \cong 0.30$}

For $Q^{2} \gtrsim 20 \mathrm{GeV}^{2}$, in both, the fit (4) and the fit (9), the $Q^{2}$ dependence of the photoabsorption cross section is given by

$$
\sigma_{\gamma^{*} p}\left(W^{2}, Q^{2}\right) \propto \frac{1}{Q^{2}} .
$$

The structure function of the proton, $F_{2}\left(x, Q^{2}\right) \cong$ $Q^{2} \sigma_{\gamma^{*} p}\left(W^{2}, Q^{2}\right)$, accordingly only depends on $W^{2}$,

$$
F_{2}\left(x, Q^{2}\right)=F_{2}\left(W^{2}=\frac{Q^{2}}{x}\right) .
$$

Explicitly, this $W$ dependence of the experimental data is shown in Fig. 1 taken from Ref. [7]. A eye-ball twoparameter fit to the experimental data in Fig. 1 gives [7]

$$
F_{2}\left(W^{2}\right)=f_{2}\left(\frac{W^{2}}{1 \mathrm{GeV}^{2}}\right)^{C_{2}},
$$

where $f_{2} \cong 0.063$ and $C_{2}=0.29$.

The observed $W^{2}$ dependence (20) of the structure function $F_{2}=F_{2}\left(W^{2}\right)$, interpreted as a consequence of the perturbative-QCD (pQCD) improved parton model, implies a remarkable constraint [7] on the magnitude of the exponent $C_{2}$.

In the $\mathrm{pQCD}$ improved parton model, the longitudinal structure function $F_{L}\left(x, Q^{2}\right)=F_{2}\left(x, Q^{2}\right) /(1+1 / R)$, where $R$ refers to the longitudinal-to-transverse photoabsorptioncross-section ratio, at a rescaled value $x \rightarrow \xi_{L} x$, for a wide range of different gluon distributions, is proportional to the

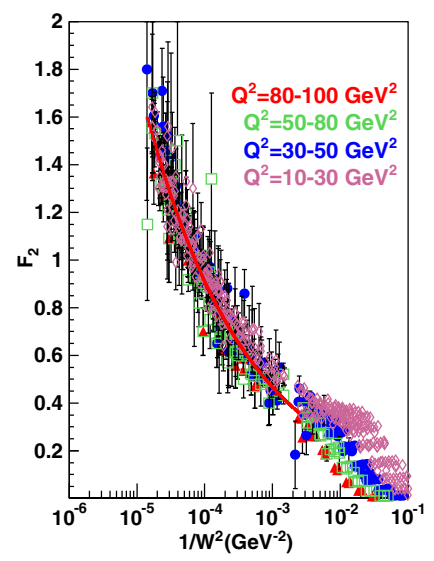

(a)

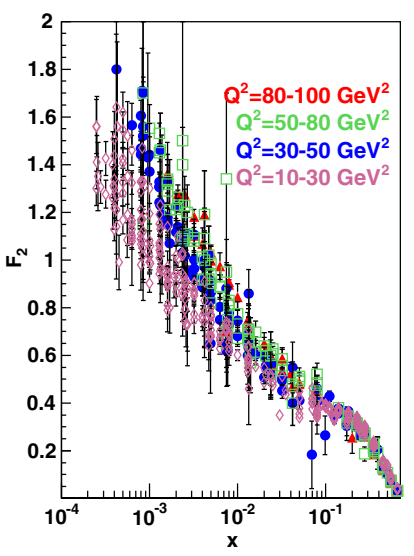

(b)
FIG. 1. In Fig. 1(a), we show the experimental data for $F_{2}(x \cong$ $\left.Q^{2} / W^{2}, Q^{2}\right)$ as a function of $1 / W^{2}$, and in Fig. 1(b), for comparison, as a function of $x$. The theoretical result based on (20) is also shown in Fig. 1(a).

gluon distribution multiplied by $\alpha_{s}\left(Q^{2}\right)$; compare to Ref. [8]. Similarly, the logarithmic derivative of $F_{2}\left(x, Q^{2}\right)$, at a rescaled value of $x \rightarrow \xi_{L} x$, is proportional to the gluon distribution [9]. Eliminating the gluon distribution, and adopting the empirically supported dependence (20) of $F_{2}=F_{2}\left(W^{2}\right)$, one finds an evolution equation for $F_{2}\left(W^{2}\right)$ that is given by [7]

$$
\left(2 \rho_{W}+1\right) \frac{\partial}{\partial \ln W^{2}} F_{2}\left(\frac{\xi_{L}}{\xi_{2}} W^{2}\right)=F_{2}\left(W^{2}\right) .
$$

In (21), we have replaced the longitudinal-to-transverse ratio of the photoabsorption cross sections via $R=1 / 2 \rho_{W}$; compare to (17).

Inserting $F_{2} \propto\left(W^{2}\right)^{C_{2}}$, see (20), from (21), we obtain the constraint [7],

$$
\left(2 \rho_{W}+1\right) C_{2}\left(\frac{\xi_{L}}{\xi_{2}}\right)^{C_{2}}=1
$$

The constraint (22) connects the exponent $C_{2}$ of the powerlaw dependence (20) of $F_{2}=F_{2}\left(W^{2}\right)$ with the value of $\rho_{W}$ that quantifies the deviation from helicity independence, $\rho_{W}=1$, of $(q \bar{q})^{J=1}$ scattering from the proton according to (14). Constancy of $C_{2}$, according to (22), implies constancy of $\rho_{W}=\rho$. From the point of view of pQCD, the constancy ${ }^{3}$ of $\rho$ implies [7] a gluon distribution multiplied by $\alpha_{s}\left(Q^{2}\right)$ that coincides with the $q \bar{q}$ sea distribution.

\footnotetext{
${ }^{3} \mathrm{~A}$ refined analysis [7] of the CDP leads to a correction of $R=1 / 2 \rho$ that implies a decrease of $R \propto 1 / \eta\left(W^{2}, Q^{2}\right)=$ $\Lambda_{\text {sat }}^{2}\left(W^{2}\right) / Q^{2}$ in the limit of very large values of $Q^{2}$ that reach the limit of $x \cong Q^{2} / W^{2} \rightarrow 0.1$.
} 
TABLE I. The value of $\rho$ determines the ratio of the cross sections for transversely, $(q \bar{q})_{T}^{J=1}$, and longitudinally polarized, $(q \bar{q})_{L}^{J=1}$, states on the proton; see (14). A value of $\rho \neq 1$ corresponds to a deviation of $R$ from the value of $R=0.5$ corresponding to helicity independence of $(q \bar{q})^{J=1} p$ scattering. Theoretical values of $C_{2}$ from (23) are compared with experimental ones. For $\left.C_{2}\right|_{\text {Exp }}=0.27 \pm 0.1$, see (30) below.

\begin{tabular}{llccc}
\hline \hline$\rho$ & $R=\frac{1}{2 \rho}$ & $\frac{1}{2 \rho+1}$ & $C_{2}$ & $\left.C_{2}\right|_{\operatorname{Exp}}$ \\
\hline 1 & 0.5 & $\frac{1}{3} \cong 0.33$ & 0.360 & \\
$\frac{4}{3}$ & 0.375 & $\frac{3}{11} \cong 0.27$ & 0.290 & $0.30 \pm 0.01$ (Ref. [1]) \\
2 & 0.25 & $\frac{1}{5}=0.20$ & 0.209 & $0.27 \pm 0.01$ (Ref. [3]) \\
\hline \hline
\end{tabular}

Expanding the exponential in (22) (to first order in $C_{2}$ ) and solving for $C_{2}$ yields

$$
C_{2} \cong \frac{1}{2 \rho+1} \frac{1}{\left(1-\frac{1}{2 \rho+1} \ln \frac{\xi_{2}}{\xi_{L}}\right)}
$$

The ratio of $\xi_{2} / \xi_{L}$ in (22) and (23) is given by $[8,9]$ $\xi_{2} / \xi_{L}=1.25$.

In Table I, ${ }^{4}$ we show the results for $C_{2}$ from (23) for different values of $\rho$ and $R=1 / 2 \rho$. We comment on the results in Table I as follows:

(i) The different dependence on the configuration, $z(1-z)$, of longitudinally and transversely polarized $(q \bar{q})^{J=1}$ states, via the uncertainty principle, implies an enhanced transverse size of transversely polarized relative to longitudinally polarized $(q \bar{q})^{J=1}$ states. This estimate within the CDP implies $\rho=4 / 3$ [7]. Consistency of the CDP with pQCD, from (23), predicts $C_{2}=0.29$, in agreement with the observed value of $\left.C_{2}\right|_{\text {Exp }}=\epsilon_{0} \cong 0.30$ from (2) and $\left.C_{2}\right|_{\text {Exp }}=$ $0.28 \pm 0.06$ from (8) and $\left.C_{2}\right|_{\text {Exp }}=0.27 \pm 0.01$ from (30) below. We conclude that the consistency between the CDP and $\mathrm{pQCD}$ is empirically established.

(ii) A value of $\rho=1$, assuming helicity independence of $(q \bar{q})^{J=1} p$ scattering, in the approximation of ignoring the transverse-size enhancement discussed in i), according to the consistency condition (23) implies $C_{2}=0.360$, which is excluded by the measured

\footnotetext{
${ }^{4}$ The "rigorous upper limit" of $R \leq 0.37248$ from the "standard color-dipole model of low x DIS" $[1,6]$, excluding $\rho=1(R=$ $1 / 2$ ) and even $\rho=4 / 3=0.375$ in Table I, depends [10] on the ad hoc assumption contained in the "standard color-dipole model" $[1,6]$ of excluding [6] a $z(1-z)$ dependence of the $(q \bar{q}) p$ cross section $\sigma_{(q \bar{q}) p}$ in (11), compare footnote 2. Fits to experimental data test this underlying assumption. A violation of the "rigorous upper limit" by experimental data can be taken care of by allowing for a dependence on $z(1-z)$; see, e.g., the ansatz (28) that implies $\rho=1$ [i.e., helicity independence of $(q \bar{q})^{J=1} p$ scattering, compare (14)] and $R=1 / 2 \rho=0.5$ in violation of the bound.
}

value of $\left.C_{2}\right|_{\operatorname{Exp}}=\epsilon_{0} \cong 0.30$. Concerning the direct experimental determination of $R=1 / 2 \rho$, see below.

(iii) A value of a transverse-to-longitudinal enhancement by a factor of $\rho=2$, or $R=1 / 2 \rho=0.25$ requires $C_{2}=\epsilon_{0}=0.21$, and is definitely excluded by $\left.C_{2}\right|_{\text {Exp }} \cong 0.30$.

(iv) We add a comment on the direct measurement of the ratio $R=1 / 2 \rho$ for $Q^{2} \gg 0$. First results from the $\mathrm{H} 1$ and ZEUS Collaborations [11,12] showed agreement [7] with the prediction of

$$
\begin{aligned}
F_{L} & =\frac{1}{1+\frac{1}{R}} F_{2}=\frac{1}{1+2 \rho} F_{2}=0.27 F_{2}, \\
(\rho & =4 / 3) .
\end{aligned}
$$

More recent measurements showed a smaller value of $\mathrm{R}$ [13]. The more indirect determination of $R$ from the two-Pomeron fit to the reduced cross sections led to values roughly between $R=0.35$ and $R=0.5$ [1]. It is very unfortunate that precision measurements on this very important ratio $R$ will not become available in the near future.

\section{LOW VALUES OF $Q^{2}$, THE PHOTOPRODUCTION LIMIT OF $Q^{2} \rightarrow 0$}

We come to the fourth part of this article, the examination of the low- $Q^{2}$ region of $Q^{2} \lesssim 20 \mathrm{GeV}^{2}$, including the photoproduction limit of $Q^{2}=0$.

In the tensor-Pomeron model, with decreasing $Q^{2}$, the contribution of the soft Pomeron (plus $f_{2 R}$ Reggeon) to the total photoabsorption cross section becomes increasingly important. In the $Q^{2}=0$ photoproduction limit, the hard Pomeron yields [1] a vanishing contribution. The softPomeron contribution behaves as [1]

$$
\sigma_{\gamma^{*} p}\left(W^{2}, Q^{2}\right) \propto\left(W^{2}\right)^{\epsilon_{1}}, \quad \text { for }\left(Q^{2} \rightarrow 0\right),
$$

where

$$
\epsilon_{1}=0.0935\left(\begin{array}{l}
+76 \\
-64
\end{array}\right) \cong 0.094
$$

close to $\epsilon_{1}=0.096$ from Ref. [2].

In the CDP, with decreasing $Q^{2} \rightarrow 0$ or increasing $1 / \eta\left(W^{2}, Q^{2}\right)$, the photoabsorption cross section receives contributions from an increasingly larger dipole size $\vec{r}_{\perp}^{\prime 2}$. The photoabsorption cross section (11) is determined by the $\vec{r}_{\perp}^{2} \rightarrow \infty$ saturation limit (15) of the dipole cross section (12). A detailed examination, based on (15), yields [7] 


$$
\begin{aligned}
\sigma_{\gamma^{*} p}\left(W^{2}, Q^{2}\right) \propto & \sigma_{T}^{(\infty)}\left(W^{2}\right) \ln \frac{\Lambda_{\mathrm{sat}}^{2}\left(W^{2}\right)}{Q^{2}+m_{0}^{2}}, \\
& \left(Q^{2}+m_{0}^{2} \ll \Lambda_{\mathrm{sat}}^{2}\left(W^{2}\right)\right) .
\end{aligned}
$$

The transition from $Q^{2} \gg \Lambda_{\text {sat }}^{2}\left(W^{2}\right)$ in (17) to $Q^{2} \ll$ $\Lambda_{\text {sat }}^{2}\left(W^{2}\right)$ in (27) occurs by a transition from $\sigma_{\gamma^{*} p}\left(W^{2}, Q^{2}\right) \propto 1 / \eta\left(W^{2}, Q^{2}\right)$ in (17) to $\sigma_{\gamma^{*} p}\left(W^{2}, Q^{2}\right) \propto$ $\ln \left(1 / \eta\left(W^{2}, Q^{2}\right)\right)$ in (27). In distinction from the twoPomeron model, no independent additional parameter must be introduced in this smooth transition from $Q^{2} \gg$ $20 \mathrm{GeV}^{2}$ to $Q^{2}=0$. The cross section (27) fulfills [7] the Froissart bound $\sigma_{\gamma^{*}} \propto \ln ^{2} W^{2}$. $^{5}$

The considerations on the CDP so far were exclusively obtained by analyzing the general expressions (11) and (12). A detailed interpolation between the limits of $1 / \eta\left(W^{2}, Q^{2}\right)$ for $\eta\left(W^{2}, Q^{2}\right) \gg 1$, and $\ln \left(1 / \eta\left(W^{2}, Q^{2}\right)\right)$ for $\eta\left(W^{2}, Q^{2}\right) \lesssim 1$, requires a specific ansatz for the dipole cross section in (11). The fit in Ref. [3] was based on the ansatz,

$$
\begin{aligned}
& \sigma_{(q \bar{q}) p}\left(\vec{r}_{\perp}, z(1-z), W^{2}\right) \\
& \quad=\sigma^{(\infty)}\left(W^{2}\right)\left(1-J_{0}\left(r_{\perp} z(1-z) \Lambda_{\text {sat }}^{2}\left(W^{2}\right)\right)\right),
\end{aligned}
$$

where $\sigma^{(\infty)}\left(W^{2}\right)$ is of hadronic size and logarithmically dependent on $W^{2}$. The ansatz (28) contains color transparency (13) and saturation (15). The dependence on $r_{\perp}^{\prime}=r_{\perp} z(1-z)$ implies [7] $\rho=1$ and $R=1 / 2 \rho=1 / 2$ for $Q^{2} \gg 0 .^{6}$

The fit to the total photoabsorption cross section (11) upon insertion of (28) gave [3]

\footnotetext{
${ }^{5}$ The photon makes a transition to (quasiasymptotic) on shell $(q \bar{q})^{J=1}$ states; see, e.g., Ref. [14] for a concise representation of this point. For, e.g., $Q^{2} \rightarrow 0$, the range of masses of contributing $(q \bar{q})^{J=1}$ states is essentially given by transitions $\gamma \rightarrow(q \bar{q})^{J=1}$ to low-lying $(q \bar{q})^{J=1}$ on shell color-dipole states representing the low-lying vector mesons, $\gamma \rightarrow\left(\rho^{0}, w, \phi\right)$. (For an early representation of the $\left(\rho^{0}, w, \phi\right)$ contributions by a massive $J=1$ continuum compare Ref. [5].) Accordingly, $\sigma_{\gamma p}\left(W^{2}\right) \propto$ $\sigma_{\left(\rho^{0}, w, \phi\right) p}\left(W^{2}\right) \propto \ln ^{2} W^{2}$, where the second step rests on the $\ln ^{2} W^{2}$ dependence of the hadronic cross section $\sigma_{\left(\rho^{0}, w, \phi\right)}\left(W^{2}\right)$ in the high-energy limit. [15]. In (27) and in (28), we have $\sigma^{(\infty)}\left(W^{2}\right) \propto \ln W^{2}$. Compare also Ref. [14] for a discussion of the $W$ dependence of $\sigma^{(\infty)}\left(W^{2}\right)$ and its experimental determination. The fact that $\gamma^{*}$ is not an asymptotic state [1] is recognized as becoming irrelevant with respect to the high-energy limit of the photoabsorption cross section as soon as the underlying (empirically supported [3,5]) dynamical mechanism of $\gamma^{*} \rightarrow$ $(q \bar{q})^{J=1}$ with subsequent on shell hadronic $(q \bar{q})^{J=1} p$ scattering is taken into account. For successful fits to the measured total cross sections for $\gamma^{*} p$, as well as $\gamma p, \pi^{ \pm} p, p p, \bar{p} p$, scattering, based on Froissart-bound saturation $\left(\ln ^{2} W^{2}\right)$ rather than Regge theory $\left(\left(W^{2}\right)^{\alpha_{j}(0)-1}\right)$, compare Ref. [16].

${ }^{6} \mathrm{~A}$ refined ansatz [7] for the dipole interaction incorporates $\rho=4 / 3$.
}

$\Lambda_{\mathrm{sat}}^{2}\left(W^{2}\right)=C_{1}\left(\frac{W^{2}+W_{0}^{2}}{1 \mathrm{GeV}^{2}}\right)^{C_{2}} \simeq C_{1}\left(\frac{W^{2}}{1 \mathrm{GeV}^{2}}\right)^{C_{2}}$,

where

$$
\begin{aligned}
& C_{2}=0.27 \pm 0.01, \\
& C_{1}=0.34 \pm 0.05 \mathrm{GeV}^{2},
\end{aligned}
$$

as well as

$$
\begin{aligned}
& m_{0}^{2}=0.16 \pm 0.01 \mathrm{GeV}^{2}, \\
& W_{0}^{2}=882 \pm 246 \mathrm{GeV}^{2} .
\end{aligned}
$$

One finds $2 \mathrm{GeV}^{2} \lesssim \Lambda_{\text {sat }}^{2}\left(W^{2}\right) \lesssim 7 \mathrm{GeV}^{2}$ for the HERA energy range of $30 \mathrm{GeV} \lesssim W \lesssim 300 \mathrm{GeV}$. The result (30) is consistent with, and improves the accuracy of the phenomenological fit (8), and it is consistent with the tensor-Pomeron fit (2). For the fit, the hadronic cross section $\sigma^{(\infty)}\left(W^{2}\right)$ was consistently expressed [3,7] in terms of a fit to the $Q^{2}=0$ photoproduction cross section. In addition to this input of a fit to the $Q^{2}=0$ photoproduction cross section, essentially only three independent fit parameters $C_{2}, C_{1}$, and $m_{0}^{2}$, have been used for a successful representation of the body of the experimental data on lowx DIS, including the transition to $Q^{2}=0$ photoproduction. Compare to the previous section for a theoretical prediction of the value of $C_{2}$.

\section{CONCLUSIONS}

We end with the following conclusions:

(i) The hard-Pomeron-fit result [1] of $\sigma_{\gamma^{*} p}\left(W^{2}, Q^{2}\right) \propto$ $\left(W^{2}\right)^{\epsilon_{0}} /\left(Q^{2}\right)^{\eta_{0}}$ for $Q^{2} \gtrsim 20 \mathrm{GeV}^{2}$, where $\epsilon_{0}=$ $0.3008\left(\begin{array}{c}+73 \\ -84\end{array}\right) \cong 0.30 \pm 0.1$ and $\eta_{0}=0.967(73) \cong$ $1.00 \pm 0.04$, based on the assumption of a powerlike $W^{2}$ dependence and 17 parameters for a modelindependent parametrization of the $Q^{2}$ dependence, confirms the prediction [3] of the CDP of $\sigma_{\gamma^{*} p}\left(W^{2}, Q^{2}\right) \propto 1 / \eta\left(W^{2}, Q^{2}\right)=\Lambda_{\text {sat }}^{2}\left(W^{2}\right) / Q^{2}$ with the predicted $1 / Q^{2}$ dependence and the fitted $W^{2}$ dependence of $\Lambda_{\text {sat }}^{2}\left(W^{2}\right) \propto\left(W^{2}\right)^{C_{2}}$, where $C_{2}=$ $0.27 \pm 0.01$.

(ii) Requiring consistency of the $\mathrm{CDP}$ and $\mathrm{pQCD}$ for sufficiently large $Q^{2} \gtrsim 10 \mathrm{GeV}^{2}$ yields the prediction of $C_{2}=0.29$ for the exponent $C_{2}$ that is confirmed by the CDP fit result of $C_{2}=0.27 \pm 0.01$ and the hard-Pomeron-fit result of $\epsilon_{0} \cong 0.30 \pm 0.1$.

(iii) In distinction from the tensor-Pomeron approach, in the CDP, the transition from $Q^{2} \gtrsim 20 \mathrm{GeV}^{2}$ to $Q^{2} \lesssim 20 \mathrm{GeV}^{2}$, including the $Q^{2}=0$ photoproduction limit, is obtained by a smooth transition from $\sigma_{\gamma^{*} p} \propto 1 / \eta\left(W^{2}, Q^{2}\right)$ to $\sigma_{\gamma^{*} p} \propto \ln \left(1 / \eta\left(W^{2}, Q^{2}\right)\right)$, without the necessity for introducing an additional fit parameter. 
(iv) The essential new element of DIS compared to photoproduction consists of the additional degree of freedom of the virtuality, $Q^{2}$, of the (virtual) photon. The $Q^{2}$ dependence of the photoabsorption cross section in the CDP is uniquely predicted as a consequence of the mass-dispersion relation of generalized vector dominance (GVD) formulated some 50 years ago. In the tensor-Pomeron-Regge approach, this $Q^{2}$ dependence is fitted by a large number of parameters to allow for a representation of the energy dependence of the photoabsorption experimental data in terms of a linear superposition of a power-law ansatz associated with, respectively, a soft and a hard Pomeron trajectory. Additional independent empirical information on the (hard) Pomeron trajectory is lacking so far, however.

\section{ACKNOWLEDGMENTS}

The author thanks Kuroda-san for useful remarks on the preliminary version of this paper.
[1] D. Britzger, C. Ewerz, S. Glazov, O. Nachtmann, and S. Schmitt, Phys. Rev. D 100, 114007 (2019).

[2] A. Donnachie and P. V. Landshoff, Phys. Lett. B 437, 408 (1998); Eur. Phys. J. C 77, 524 (2017).

[3] D. Schildknecht, Nucl. Phys. B, Proc. Suppl. 99, 121 (2001); D. Schildknecht, B. Surrow, and M. Tentyukov, Phys. Lett. B 499, 116 (2001); G. Cvetic, D. Schildknecht, B. Surrow, and M. Tentyukov, Eur. Phys. J. C 20, 77 (2001); D. Schildknecht, Acta Phys. Pol. B 37, 595 (2006).

[4] G. Cvetic, D. Schildknecht, and A. Shoshi, Eur. Phys. J. C 13, 301 (2000).

[5] J. J. Sakurai and D. Schildknecht, Phys. Lett. 40B, 121 (1972); B. Gorczyca and D. Schildknecht, Phys. Lett. 47B, 71 (1973); H. Fraas, B. J. Read, and D. Schildknecht, Nucl. Phys. B86, 346 (1975); R. Devenish and D. Schildknecht, Phys. Rev. D 14, 93 (1976).

[6] C. Ewerz and O. Nachtmann, Ann. Phys. (Amsterdam) 322, 1670 (2007).

[7] M. Kuroda and D. Schildknecht, Phys. Rev. D 85, 094001 (2012); M. Kuroda and D. Schildknecht, Int. J. Mod. Phys. A 31, 1650157 (2016).
[8] A. D. Martin, R. G. Roberts, and W. J. Stirling, Phys. Rev. D 37, 1161 (1988); A. M. Cooper-Sarkar et al., Z. Phys. C 39, 281 (1988).

[9] K. Prytz, Phys. Lett. B 311, 286 (1993).

[10] D. Schildknecht, Phys. Lett. B 716, 413 (2012).

[11] F. D. Aaron et al. (H1 Collaboration), Phys. Lett. B 665, 139 (2008).

[12] S. Chekanov et al. (ZEUS Collaboration), Phys. Lett. B 682 , 8 (2009).

[13] V. Andreev et al. (H1 Collaboration), Eur. Phys. J. C 74, 2814 (2014).

[14] M. Kuroda and D. Schildknecht, Phys. Rev. D 96, 094013 (2017), Appendix A.

[15] W. Heisenberg, in Vorträge über Kosmische Strahlung (Springer, Berlin 1953), p. 155, reprinted in W. Heisenberg, Collected Works, Series B (Springer Berlin 1984), p. 498; W. Heisenberg, Die Naturwissenschaften 61, 1 (1974), reprinted in Collected Works, Series B, p. 912; M. Froissart, Phys. Rev. 123, 1053 (1961).

[16] M. M. Block, E. L. Berger, and C.-I. Tan, Phys. Rev. Lett. 97, 252003 (2006); Edmund L. Berger, M. M. Block, and C.-I. Tan, Phys. Rev. Lett. 98, 242001 (2007). 